\title{
RELACIÓN ENTRE LA AUTOESTIMA Y LA SATISFACCIÓN CON LA VIDA EN UNA MUESTRA DE ESTUDIANTES UNIVERSITARIOS
}

\author{
Paula Ruiz-González \\ Laboratorio de Inteligencia Emocional, Universidad de Cádiz (Cádiz, España) \\ Instituto de Investigación para el Desarrollo Social Sostenible (INDESS) Jerez de la Frontera, Universidad de \\ Cádiz (Cádiz, España) \\ paula.ruiz@uca.es \\ Yolanda Medina-Mesa \\ Centro de Psicología Yolanda Medina, Jerez (Cádiz, España) \\ Antonio Zayas \\ Laboratorio de Inteligencia Emocional, Universidad de Cádiz (Cádiz, España) \\ Instituto de Investigación para el Desarrollo Social Sostenible (INDESS) Jerez de la Frontera, Universidad de \\ Cádiz (Cádiz, España) \\ Rocío Gómez-Molinero \\ Laboratorio de Inteligencia Emocional, Universidad de Cádiz (Cádiz, España)
}

Fecha de Recepción: 16 Febrero 2018

Fecha de Admisión: 10 Abril 2018

\section{RESUMEN}

Las nuevas exigencias y cambios transicionales durante la etapa universitaria pueden suponer una fuente de estrés en el alumnado repercutiendo notablemente sobre su bienestar (Gutiérrez et al., 2010). El propósito de este estudio fue analizar la capacidad predictiva de la autoestima sobre la satisfacción con la vida -componente cognitivo del bienestar subjetivo-. La muestra estuvo conformada por 150 estudiantes ( $72 \%$ mujeres, $28 \%$ hombres) pertenecientes a diferentes grados de la Universidad de Cádiz cuya media de edad fue 21,62 ( $S D=3.59$, rango=18-48). Se administró la Escala de Autoestima de Rosenberg -RSES- (Rosenberg, 1965; adaptación al castellano de Salgado e Iglesias, 1995) y la Escala de Satisfacción con la Vida -SWLS- (Diener, Emmons, Larsen y Griffin, 1985; adaptación al castellano de Atienza, Pons, Balaguer y García-Merita, 2000). Los análisis de regresión jerárquica realizados indicaron que todas las variables introducidas explicaban el 34,4\% de la varianza de la satisfacción con la vida $\left(R^{2}=.34\right)$, si bien el $32,6 \%$ se debía a la influencia de la autoestima, siendo su capacidad predictiva positiva y estadísticamente significativa $(\beta=.61)$. Estos resultados se confirmaron con independencia del posible efecto de la edad, el sexo y la titulación cursada por el alumnado universitario. Estos datos aportan apoyo empírico sobre la importancia de desarrollar programas que refuercen la autoestima de estudiantes universitarios, pues simultáneamente se incrementarían los niveles de satisfacción global con la vida. 
Palabras clave: satisfacción con la vida; autoestima; bienestar subjetivo; universitarios

\section{ABSTRACT}

Relationship between self-esteem and life satisfaction in a sample of university students.

New demands and transitional changes during university stage can be a source of stress for university students, having an important effect on their well-being (Gutiérrez et al., 2010). The purpose of this study was to analyze the predictive ability of self-esteem on life satisfaction -cognitive component of subjective well-being-. The sample consisted of 150 students ( $72 \%$ women, $28 \%$ men) attending to different degrees at the University of Cadiz and whose average age was 21.62 (SD = 3.59, range $=18-48$ ). The Rosenberg Self-Esteem Scale -RSES- (Rosenberg, 1965; Spanish version by Salgado \& Iglesias, 1995) and the Life Satisfaction Scale -SWLS- (Diener, Emmons, Larsen \& Griffin, 1985; Spanish version by Atienza, Pons, Balaguer \& García-Merita, 2000) were administered. Hierarchical regression analysis carried out indicated that all the variables entered explained $34.4 \%$ of the variance in life satisfaction $\left(R^{2}=.34\right)$, although $32,6 \%$ was due to the influence of selfesteem, being its predictive ability positive and statistically significant $(\beta=.61)$. These results were confirmed independently of the possible effect of age, sex, and students' degree. These data provide empirical support concerning the importance of developing programs that reinforce students' selfesteem enhancing, simultaneously, global life satisfaction levels.

Keywords: life satisfaction; self-esteem; subjective wellbeing; university students

\section{ANTECEDENTES}

A pesar de que la Psicología a lo largo de su evolución se ha focalizado en la concepción clásica de patología, el creciente interés por la Psicología Positiva y el surgimiento de nuevos modelos centrados en el desarrollo positivo, ha dado lugar a numerosas investigaciones centradas en los beneficios de la adaptación psicológica (Guil, Zatas, Gil-Olarte, Guerrero, González y Mestre, 2016; Reina, Oliva y Parra, 2010; San Martín y Barra, 2013). Esta visión trata de conocer qué factores promueven un desarrollo saludable desde una perspectiva integral y holística que facilite una mejor comprensión del comportamiento humano (Rodríguez, Ramos, Ros, Fernández y Revuelta, 2016).

Dentro de este paradigma, conceptos como el bienestar están recibiendo una considerable atención por parte de los profesionales de la psicología (Rodríguez y Goñi, 2011). El bienestar es un componente de la salud manifestado en conductas dirigidas al mantenimiento de la misma tanto física como mentalmente, y que influye en el funcionamiento de sistemas y órganos (OMS, 2003; Montoya y Landero, 2008).

A partir de este concepto surge el de bienestar subjetivo, referido a la evaluación global que se hace de la vida y manifestado en todas las áreas de la actividad humana (Atienza, Pons, Balaguer y García-Merita, 2000; Taylor, 1991). Pavot y Diener (2013) aportan una concepción más completa considerándolo el resultado de dos componentes en estrecha interrelación: el componente emocional (Lucas, Diener y Suh, 1996; Sandín, 1999), caracterizado por afectividad positiva o negativa; y el componente cognitivo, denominado satisfacción con la vida (Andrews y Withey, 1976).

La satisfacción con la vida es definida por Undurraga y Avendaño (1998) como el sentimiento de bienestar en relación consigo mismo en la propia vida, diferenciándose de la aprobación-desaprobación o del grado de satisfacción con las condiciones objetivas de vida. Se trata de una evaluación de la calidad de vida de manera global y de acuerdo a un criterio propio (Pavot y Diener, 1993).

Estudios realizados con población universitaria han mostrado como la satisfacción con la vida depende del logro de objetivos a lo largo de la trayectoria vital y del apoyo social recibido (Castro y Sánchez, 2000; Novoa y Barra, 2015). Asimismo, ha correlacionado con autoaceptación, dominio del entorno y propósito en la vida (Blanco y Díaz, 2005; Zubieta y Delfino, 2010). 
La importancia de estudiar y potenciar la satisfacción vital reside en sus beneficios sobre el funcionamiento intra e interpersonal. Además, ha mostrado una relación inversa con síntomas depresivos o la disforia de género (Chico y Ferrando, 2008; Rabito y Rodriguez, 2016). Sin embargo, quienes se encuentran extremadamente insatisfechos, muestran mayor vulnerabilidad a presentar conductas agresivas, ideación suicida, conductas sexuales de riesgo, consumo de sustancias y problemas físicos (Huebner y Diener, 2008).

Por tanto, es especialmente interesante estudiar la satisfacción vital en alumnado universitario, pues se trata de un período en el que el afrontamiento de nuevas exigencias, repercute en la percepción y valoración que los estudiantes hacen de sí mismos. Es posible inferir que la existencia de diferentes fuentes de estrés durante esta etapa dificulte el afrontamiento de situaciones nuevas provocando que su satisfacción vital se vea mermada (Novoa y Barra, 2015). Su interés de estudio pasaría por examinar qué variables propician una mayor satisfacción vital y el efecto moderador que puedan establecer sobre el bienestar de los estudiantes. Entre ellas, la autoestima ha mostrado una fuerte influencia sobre la satisfacción con la vida (Borges, Ruiz, Salar y Moreno, 2016; Reina et al., 2010; San Martín y Barra, 2013).

La autoestima es definida por Rosenberg (1965) como una actitud integral que tiene una persona hacia sí misma, ya sea de carácter positivo o negativo. Referida a la confianza en el propio potencial (Undurraga y Avendaño, 1998), se caracteriza por dos componentes: el sentimiento de capacidad para alcanzar el éxito y la valía personal (Tarazona, 2005).

Elevados niveles de autoestima se han vinculado con una mayor adaptación social, felicidad y menor vulnerabilidad a presentar conductas de riesgo (Guterman, Hahm y Cameron, 2002; Parra, Oliva, y Sánchez-Queija, 2004; Ramírez, 2018). En el contexto universitario se ha relacionado con la permanencia escolar, mayor bienestar emocional y mejor desempeño laboral futuro (Chaves et al., 2013; San Martín y Barra, 2013). Por el contrario, bajos niveles se relacionan con valoraciones negativas de la vida, dificultades de adaptación a situaciones adversas y mayor riesgo de conducta suicida (Furr y Fander, 1998; Montes y Tomás, 2016).

A pesar de que la autoestima no es la variable que mejor predice la satisfacción con la vida, explica gran variabilidad de la misma (San Martín y Barra, 2013). Numerosos trabajos indican que niveles altos de autoestima predicen una mayor satisfacción vital (Reina et al., 2010; Rodríguez et al., 2016), mientras que una baja autoestima se relaciona con valoraciones negativas de la vida (Furr y Fander, 1998).

La autoestima actuaría como amortiguador cognitivo de la satisfacción vital al atenuar el impacto psicológico provocado por experiencias negativas (Cummins, 2010). Aquellos que poseen baja autoestima pueden ver afectados sus niveles de satisfacción vital ante eventos negativos, ya que amenazarían al bienestar personal de forma más intensa.

Al mismo tiempo, Cummins y Nistico (2002) postulan que elevados niveles de autoestima contribuyen a una mayor estabilidad en el tiempo en reportes de satisfacción vital. Sin embargo, se ha demostrado que esta relación está sujeta a diferencias culturales, siendo más fuerte en culturas individualistas (Kang, Shaver, Sue, Min y Jing, 2003).

En cuanto a la relación que estas variables tienen con el sexo de los estudiantes, se han encontrado resultados dispares. Mientras que ciertos estudios indican que no existen diferencias significativas entre ambos sexos (Ash y Huebner, 2001; Gilman y Huebner, 2003; Kong, Ding y Zhao, 2015; Matud, Ibáñez, Marrero y Carballeira, 2003), otros han encontrado ligeras diferencias a favor del sexo masculino en la satisfacción con la propia vida (Goldbeck, Schmitz, Besier, Herschbach y Herich, 2007; Reina et al., 2010, Reina y Oliva, 2015) y puntuaciones más bajas de autoestima en las mujeres (Chabrol et al., 2004; Khanlou, 2004; Povedano, Hendry, Ramos y Varela, 2011; Reina et al., 2010). 
Respecto a diferencias de edad, ciertas investigaciones confirman un descenso de la autoestima durante la adolescencia temprana que tiende a estabilizarse en años posteriores (Parra et al., 2004). Además, los aspirantes a la universidad se evalúan más e inseguros e incapaces de alcanzar el éxito que los alumnos que se encuentran cursando un grado universitario (González, Valdez y Serrano, 2003).

Por el contrario, la satisfacción vital se caracteriza por una mayor estabilidad, pese a ciertos declives acaecidos durante la adolescencia (Huebner, Drane y Valois, 2000; Reina et al., 2010). La valoración de la vida durante la etapa universitaria viene determinada principalmente por las oportunidades provenientes de la familia, el gobierno y la vida en general (Noriega, 2001).

No obstante, es importante conocer la influencia que puedan ejercer otras variables sobre la autoestima y la satisfacción vital. Atendiendo a la revisión de la literatura, es evidente la escasez de estudios que relacionan estas variables con la titulación. Lledó, Perandones, Herrera y Lorenzo (2014) informan no haber encontrado diferencias significativas en estas variables entre estudiantes pertenecientes a diferentes carreras universitarias. Sin embargo, se han hallado ligeras diferencias en satisfacción con la vida considerando la universidad de procedencia (Moreta, Gabior y Barrera, 2017).

Dada la influencia que pueden ejercer factores demográficos, se cree conveniente incluir la edad, el sexo y la titulación como variables control. Nuestro principal objetivo será analizar la capacidad predictiva de la autoestima sobre la satisfacción vital, controlando la posible influencia que pueda ejercer el sexo, la edad y la titulación de estudiantes universitarios. Se llevará a cabo un análisis de regresión jerárquica.

\section{PARTICIPANTES}

En el presente estudio participaron un total de 150 estudiantes pertenecientes a la Universidad de Cádiz con edades comprendidas entre 18 y 48 años ( $M=21,62 ; D T=3,59)$. El $72 \%$ de la muestra eran mujeres. Los participantes pertenecían a titulaciones de Psicología $(56,7 \%)$, Relaciones Laborales y Recursos Humanos (39,3\%), Ciencias del Mar (2,7\%) y Educación Infantil (2\%).

Los cuestionarios fueron cedidos por docentes y alumnos colaboradores a los estudiantes en sus respectivas aulas. Dado que no existía limitación de tiempo para responderlos, podían ser rellenados en casa. Previamente, debían firmar un consentimiento donde se les informaba acerca del carácter anónimo del cuestionario, la voluntariedad del estudio y la confidencialidad y protección de datos de carácter personal.

\section{INSTRUMENTOS}

Escala de Autoestima de Rosenberg -RSES- (Rosenberg, 1965; adaptación al castellano de Salgado e Iglesias, 1995). Desarrollada por Rosenberg (1965) para la evaluación de la autoestima en la adolescencia, es uno de los instrumentos más utilizados para la medición global de la autoestima. Evalúa los sentimientos de respeto y aceptación de uno mismo/a a través de 10 ítems con una escala de 1 a 4 puntos ( $1=$ muy en desacuerdo; $4=$ muy de acuerdo). Cinco de los ítems están redactados de forma positiva (i.e., "Creo que tengo algunas cualidades buenas"), y los otros cinco restantes de forma negativa (i.e, "Creo que no tengo mucho de lo que estar orgulloso"). Ha sido adaptada y validada en población española mostrando propiedades psicométricas satisfactorias (Salgado e Iglesias, 1995). La consistencia interna calculada mediante alfa de Cronbach es de 0.80 . En el presente estudio se obtuvo una fiabilidad de la misma de $\alpha=0.942$.

Escala de Satisfacción con la Vida -SWLS- (Diener, Emmons, Larsen y Griffin, 1985; adaptación al castellano de Atienza, Pons, Balaguer y García-Merita, 2000). Esta escala evalúa los aspectos cog- 
nitivos del bienestar. Está compuesta por cinco ítems del tipo: "Si tuviera que vivir mi vida de nuevo, no cambiaría nada" evaluados mediante escala tipo Likert de 1 a 7 (1= completamente en desacuerdo; $7=$ completamente de acuerdo). Ha mostrado obtener muy buenas propiedades psicométricas y una elevada consistencia interna, obteniendo un coeficiente alfa de Cronbach que oscila entre 0.79 y 0.89 . En el presente estudio se obtuvo una fiabilidad de $\alpha=0.863$.

\section{RESULTADOS}

En la tabla 1 se presentan las correlaciones ( $r$ de Pearson) entre las variables de este estudio. Como se aprecia, la titulación cursada mostró una correlación positiva y estadísticamente significativa tanto con la edad como con el sexo de los estudiantes. A su vez, la autoestima se asoció positiva y significativamente con el grado cursado por los estudiantes y la edad de los mismos. Esto supondría que los universitarios presentan mayores niveles de autoestima a medida que se incrementa su edad. Es así mismo positiva y significativa la correlación establecida entre la satisfacción con la vida y la autoestima de los participantes del estudio, es decir, una mayor autoestima se corresponde con mayores niveles de satisfacción con la vida. Sin embargo, no se hallaron correlaciones entre esta última y el resto de variables del estudio.

Tabla 1. Correlaciones bivariadas entre las variables del estudio.

\begin{tabular}{llllll}
\hline & 1 & 2 & 3 & 4 & 5 \\
\hline 1. Edad & - & & & \\
2. Sexo &, 156 & - & & \\
3. Titulación & $\mathbf{, 2 9 2}^{* *}$ & $\mathbf{, 2 3 2}$ & - & & \\
4. Autoestima & $\mathbf{, 2 2 1}^{* *}$ &, 129 & $\mathbf{, 2 5 5}^{* *}$ & - & \\
5. Satisfacción con la vida &,- 001 &, 066 &, 022 & $\mathbf{5 6 3}^{* * *}$ & - \\
\hline
\end{tabular}

**Las correlaciones son significativas al nivel .01 (bilateral)

Luego de realizar la prueba de correlación entre las variables, se buscó confirmar su relación causal mediante una prueba de regresión jerárquica. Se estableció la satisfacción vital como variable dependiente, mientras que la autoestima fue la variable predictora. Con objeto de controlar la influencia de variables sociodemográficas (sexo, edad y titulación), estas fueron introducidas en un primer paso. En un segundo paso fue introducida la autoestima. Los resultados del análisis de regresión jerárquica pueden observarse en la Tabla 2.

Tabla 2. Análisis de regresión jerárquica: predictores de la satisfacción vital en universitarios

\begin{tabular}{lllllll}
\hline & $F$ & $R^{2}$ & Adj. $R^{2}$ & $\beta$ & \multicolumn{1}{l}{} & $p$ \\
\hline Paso 1 & .227 & 0.005 & -0.016 & & & \\
Sexo & & & 0.066 & 0.777 & 0.438 \\
Edad & & & -0.015 & -0.169 & 0.866 \\
Titulación & & & 0.010 & 0.119 & 0.905 \\
Paso 2 & $19.006^{* * *}$ & 0.344 & 0.326 & & & \\
Sexo & & & & 0.030 & 0.428 & 0.670 \\
Edad & & & -0.109 & -1.520 & 0.131 \\
Titulación & & & -0.110 & -1.499 & 0.136 \\
Autoestima & & & 0.611 & $8.660^{* * *}$ & 0.000 \\
\hline$* * * p<0.001$ & & &
\end{tabular}


Como se aprecia en la tabla, ninguna variable sociodemográfica explicó significativamente la satisfacción con la vida en el paso 1. Lo mismo ocurrió en el paso 2, sin embargo, fue la autoestima la única variable que mostró capacidad explicativa sobre la satisfacción con la vida. Estos análisis indican que, a pesar de que todas las variables introducidas explicaron el $34.4 \%$ de la varianza de la satisfacción con la vida $\left(\mathrm{R}^{2}=.34\right)$, la autoestima explicaba por sí misma el $32,6 \%$, mostrando además una capacidad predictiva positiva y estadísticamente significativa $(p=0.000 ; p<0.001)$. Los valores de beta indican que, si se incrementa en un punto la autoestima, la satisfacción con la vida se vería mejorada en 0.61 puntos $(\beta=.61)$.

Estos resultados se confirmaron con independencia del posible efecto de la edad, el sexo y la titulación cursada por el alumnado universitario.

\section{DISCUSIÓN}

Este estudio se había propuesto como objetivo, establecer la relación entre la autoestima y la satisfacción con la vida controlando la influencia que pudieran ejercer variables sociodemográficas como la edad, el sexo y la titulación en alumnado universitario. Si bien numerosos estudios ya indicaban la existencia de una relación positiva entre la autoestima y la satisfacción con la vida, adicionalmente debían tenerse en cuenta otras variables que, incluyéndolas como control en el análisis de regresión jerárquica, pudieran tener un efecto moderador en dicha relación.

En consonancia con el análisis de regresión jerárquica realizado y hallazgos empíricos previos, se observa como las variables disposicionales como la autoestima muestran una elevada correlación y capacidad predictiva sobre la satisfacción con la vida (Barrantes y Ureña, 2015; Cummins y Nistico, 2002; Diener et al., 1999). Este resultado es coherente con la mayoría de investigaciones del ámbito psicosocial que establecen a ambas variables como estrechamente relacionadas entre sí y a la autoestima como uno de los mejores predictores del bienestar subjetivo (Martínez, Buelga y Cava, 2007).

Por el contrario, variables demográficas como la edad, el sexo y la titulación son predictores débiles de nuestra variable de interés. Que estas variables no predigan la satisfacción con la vida en nuestro estudio no supone algo novedoso, ya que previamente otras investigaciones han encontrado resultados semejantes (Headey y Wearing, 1999; Castro y Sánchez, 2000; Lledó et al., 2014). Estos resultados también se han encontrado en estudios realizados con población adolescente (Huebner y Diener, 2008; Proctor, Linley y Maltby, 2009).

Sin embargo, independientemente de la influencia que pudieran ejercer otro tipo de variables, parece obvio el impacto que ejerce la autoestima sobre la satisfacción vital, pues ésta última se vería incrementada al aumentar los niveles de autoestima de los estudiantes.

\section{CONCLUSIONES}

La relevancia de realizar estudios desde el enfoque de la psicología positiva reside en el diseño de intervenciones que, al incidir en las variables que contribuyen al aumento del bienestar subjetivo, incrementen los niveles de satisfacción vital y promuevan un mejor ajuste psicológico. La implementación de programas que exploten el potencial humano es esencial para un continuo proceso de crecimiento personal. Ello es especialmente importante en etapas como la universitaria, caracterizadas por cambios transicionales y crisis vitales.

Es evidente que la autoestima tiene un gran impacto sobre el bienestar subjetivo de un individuo. Por ello, y derivado de los resultados de este estudio, se pone de manifiesto que, dada la capacidad explicativa y predictiva de la autoestima sobre la satisfacción vital, se diseñen intervenciones que potencien la autoestima en universitarios, pues simultáneamente se incrementarán los niveles de satisfacción con la vida. 
Sin embargo, este estudio no está exento de limitaciones. Entre las más importantes se encuentra el uso de medidas de autoinforme, pues pese a la calidad psicométrica de los instrumentos utilizados, las puntuaciones pueden estar afectados por la deseabilidad social, es decir, por la tendencia de los participantes a mostrar una presentación positiva de sí mismos.

Como líneas futuras y basados en los resultados de este estudio, podría ser interesante realizar estudios longitudinales, 0 ampliar el rango de edades en estudios transversales con el fin de observar diferencias intraindividuales y/o el cambio que sufren las variables analizadas según la trayectoria evolutiva de los sujetos. Igualmente, podría ser interesante incluir en otros estudios la autoestima en sus dimensiones física, social, ética, académica y afectiva. De esta manera podrían observarse ciertas diferencias que, siendo tratada en su dimensión general, podrían pasar desapercibidas.

\section{REFERENCIAS}

Andrews, F.M. y Withey, S.B. (1976). Social indicators of well-being: America's perception of life quality. New York: Plenum Press.

Ash, C., y Huebner, E. S. (2001). Environmental events and life satisfaction reports of adolescents: A Test of Cognitive Mediation. School Psychology International, 22, 320-336.

Atienza, F. L., Pons, D., Balaguer, I., y García Merita, M. (2000). Propiedades psicométricas de la Escala de Satisfacción con la Vida en adolescentes. Psicothema, 12(2), 314-319.

Barrantes-Brais, K., y Ureña-Bonilla, P. (2015). Bienestar psicológico y bienestar subjetivo en estudiantes universitarios costarricenses. Revista Intercontinental de Psicología y Educación, 17(1).

Blanco, A y Díaz, D. (2005). El bienestar social: su concepto y medición. Psicothema, 17(4), 582589.

Borges-Silva, F., Ruiz-González, L., Salar, C., y Moreno-Murcia, J. A. (2017). Motivación, autoestima y satisfacción con la vida en mujeres practicantes de clases dirigidas en centros de fitness. Cultura, Ciencia y Deporte, 12(34).

Castro, A., y Sánchez, M. P. (2000). Objetivos de vida y satisfacción autopercibida en estudiantes universitarios. Psicothema, 12(1).

Chabrol, H., Carlin, E., Michaud, C., Rey, A., Cassan, D., Juilot, M. et al. (2004). A study of the Rosenberg self-estem scale in a sample of high-school students. Neuropsychiatrie del'Enfance et del'Adolescence, 52, 533-536.

Chaves, E. C., Simäo, T. P., de Oliveira, L. S., de Souza, I. P., Lunes, D. H. y Nogueira, D. A. (2013). Assessment of Nursing Students' Self-Esteem at a University in the South of Minas Gérais (Brazil). Investigación \& Educación en Enfermería, 31(2), 261-269.

Chico, E., y Ferrando, P. (2008). Variables cognitivas y afectivas como predictoras de satisfacción. Psicothema, 20(3), 408-412

Cummins, R. A. (2010). Fluency disorders and life quality: Subjective wellbeing vs. health-related quality of life. Journal of fluency disorders, 35(3), 161-172.

Cummins, R. A., y Nistico, H. (2002). Maintaining life satisfaction: The role of positive bias. Journal of Happiness Studies, 3, 37-69.

Furr, R.M. y Funder. D (1998). A multimodal analysis of personal negativity. Journal of Personality and Social Psychology, 74, 1580-1591.

Gilman, R. y Huebner, S. (2003). A review of life satisfaction research with children and adolescents. School Psychology Quarterly, 18, 192-205.

Goldbeck, L., Schmitz, T. G., Besier, T., Herschbach, P., y Henrich, G. (2007). Life satisfaction decreases during adolescence. Quality of Life Research, 16, 969-979.

González-Arratia, N. I., Valdez Medina, J. L., y Serrano García, J. M. (2003). Autoestima en jóvenes universitarios. CIENCIA ergo sum, 10(2). 
Guil, R., Zayas, A., Gil-Olarte, P., Guerrero, C., González, S., y Mestre, J. M. (2016). Bienestar psicológico, optimismo y resiliencia en mujeres con cáncer de mama. Psicooncología, 13(1), 127138.

Guterman, N.B., Hahm, H.C. y Cameron, M. (2002). Adolescent victimization and subsequent use of mental health counselling services. Journal of Adolescent Health, 30, 336-345.

Gutiérrez, J. A., Montoya, L. P., Toro, B. E., Briñón, M. A., Rosas Restrepo, E., y Salazar, L. E. (2010). Depresión en estudiantes universitarios y su asociación con el estrés académico. CES Medicina, 24(1), 7-17.

Headey, B. y Wearing, A. (1991). Subjective well-being: A stocks and flows framework. In F. Strack, M. Argyle, y N. Schwarz (Eds.), Subjective well-being: An interdisciplinary perspective (pp. 4173). Oxford, England: Pergamon Press.

Huebner, E. S., Drane J. W., y Valois R. F. (2000). Levels and demographic correlates of adolescent life satisfaction reports. School Psychology International, 21, 281-292.

Huebner, E. S., y Diener, C. (2008). Research on life satisfaction of children and youth: Implications for the delivery of school-related services. En M. Eid y R. Larsen (Eds.), The science of subjective well-being (pp. 376-392). Nueva York: Guilford Press.

Kang, S., Shaver, P. R., Sue, S., Min, K., y Jing, H. (2003). Culture-specific patterns in the prediction of life satisfaction: Roles of emotion, relationship quality, and self-esteem. Personality and Social Psychology Bulletin, 29, 1596 -1608.

Khanlou, N. (2004). Influences on adolescent self-esteem in multicultural Canadian schools. Public Health Nursing, 21, 404-411.

Kong, F., Ding, K., y Zhao, J. (2015). The relationships among gratitude, self-esteem, social support and life satisfaction among undergraduate students. Journal of Happiness Studies, 16(2), 477489.

Lledó Carreres, A., Perandones González, T. M., Herrera Torres, L., y Lorenzo Lledó, G. (2014). Cansancio emocional, autoestima y satisfacción con los estudios en estudiantes universitarios. International Journal of Developmental and Educational Psychology, 7(1).

Lucas, R.E., Diener, E. y Suh, E. (1996). Discriminat validity of well-being measures. Journal of Personality and Social Psychology, 71, 616-628.

Matud M. P., Ibáñez I., Marrero R. J., y Carballeira M. (2003). Diferencias en autoestima en función del género. Análisis y Modificación de Conducta, 29, 51-78.

Martínez-Antón, M., Buelga, S., y Cava, M. J. (2007). La satisfacción con la vida en la adolescencia y su relación con la auto-estima y el ajuste escolar. Anuario de Psicología, 38(29), 293-303.

Montes-Hidalgo, J., y Tomás-Sábado, J. (2016). Autoestima, resiliencia, locus de control y riesgo suicida en estudiantes de enfermería. Enfermería Clínica, 26(3), 188-193.

Montoya, B., y Landero, R. (2008). Satisfacción con la vida y autoestima en jóvenes de familias monoparentales y biparentales. Psicología y Salud, 18(1), 117-122.

Moreta, R., Gabior, I., y Barrera, L. (2017). El bienestar psicológico y la satisfacción con la vida como predictores del bienestar social en una muestra de universitarios ecuatorianos. Salud \& Sociedad, 8(2).

Noriega, J. A. V. (2001). Bienestar subjetivo en una muestra de jóvenes universitarios. Revista intercontinental de psicología y educación, 3(1), 11-21.

Novoa, C., y Barra, E. (2015). Influencia del apoyo social percibido y los factores de personalidad en la satisfacción vital de estudiantes universitarios. Terapia psicológica, 33(3), 239-245.

Organización Mundial de la Salud (2003). Informe sobre la salud en el mundo. Forjemos el futuro: atención primaria de la salud. Ginebra: OMS 
Parra, A., Oliva, A., y Sánchez-Queija, I. (2004). Evolución y determinantes de la autoestima durante los años adolescentes. Anuario de Psicología, 35, 331- 346.

Pavot, W. y Diener, E. (1993). Revisión de la satisfacción con la Escala de Satisfacción con la Vida. Gravamen Psicológico, 5(2), 164-172.

Pavot, W. y Diener, E. (2013). Happiness experienced: The science of subjective wellbeing. En S. A. David, I. Boniwell y A. Conley Ayers (Eds.), The Oxford handbook of happiness (pp. 134-151). New York, NY: Oxford University Press.

Povedano, A., Hendry, L. B., Ramos, M. J., y Varela, R. (2011). Victimización escolar: clima familiar, autoestima y satisfacción con la vida desde una perspectiva de género. Psychosocial Intervention, 20(1), 5-12.

Proctor, C. L., Linley, P. A., y Maltby, J. (2009). Youth life satisfaction: A review of the literature. Journal of Happiness Studies, 10, 583-630.

Rabito-Alcón, M., y Rodríguez-Molina, J. (2016). Satisfaction with life and psychological well-being in people with gender dysphoria. Actas Esp Psiquiatr, 44(2), 47-54.

Ramírez, M. S. 0. (2018). Calidad de vida, autoestima y felicidad en mujeres durante el climaterio, de estrato socio-económico medio-alto y bajo, en la ciudad de lima. Consensus, 17(1), 181-193.

Reina, M. D. C., y Oliva, A. (2015). From emotional competence to self-esteem and life-satisfaction in adolescents. Behavioral Psychology, 23(2), 345-359.

Reina, M.C., Oliva, A. y Parra, A. (2010). Percepciones de autoevaluación: autoestima, autoeficacia y satisfacción vital en la adolescencia. Psychology, Society \& Education, 2(1), 55-69.

Rodríguez-Fernández, A. y Goñi-Grandmontagne, A. (2011). La estructura tridimensional del bienestar subjetivo. Anales de psicología, 27(2), 327-332.

Rodríguez-Fernández, A., Ramos-Díaz, E., Ros, I., Fernández-Zabala, A., y Revuelta, L. (2016). Bienestar subjetivo en la adolescencia: el papel de la resiliencia, el autoconcepto y el apoyo social percibido. Suma psicológica, 23(1), 60-69.

Rosenberg, M. (1965). Society and the adolescent self-image. Princeton: Princeton University Press

Salgado, J. F. y Iglesias, M. (1995). Estructura factorial de la escala de autoestima de Rosenberg: Un análisis factorial confirmatorio. Psicológica, 16, 441-454.

San Martín, J. L., y Barra, E. (2013). Autoestima, apoyo social y satisfacción vital en adolescentes. Terapia psicológica, 31(3), 287-291.

Sandín, B. (1999). El estrés psicosocial: Conceptos y consecuencias clínicas. Madrid: Klinik.

Tarazona, D. (2005). Autoestima, satisfacción con la vida y condiciones de habitabilidad en adolescentes estudiantes de quinto año de media. Un estudio factorial según pobreza y sexo. Revista de Investigación en Psicología, 8(2), 57-65.

Taylor, S.E. (1991). Seamos optimistas. Ilusiones positivas. Barcelona: Martínez Roca.

Undurraga, C. y Avendaño, C. (1998). Dimensión psicológica de la pobreza. Psykhe, 6(1), 57-63.

Zubieta, E. M., y Delfino, G. (2010). Satisfacción con la vida, bienestar psicológico y bienestar social en estudiantes universitarios de Buenos Aires. Anuario de investigaciones, 17, 277-283. 
